\title{
The Role of Capillary Pressure in Compositional Simulation of Heterogeneous Reservoirs
}

\author{
Barker J. W., Sindic G.
}

Elf Geoscience Research Centre, U. K.

\begin{abstract}
Copyright 1996, Steering Committee of the European IOR - Symposium.
This paper was presented at the 8th. European IOR - Symposium in Vienna, Austria, May 16 - 17, 1996

This paper was selected for presentation by the Steering Committee, following review of information contained in an abstract

submitted by the author(s). The paper, as presented has not been reviewed by the Steering Committee.
\end{abstract}

\begin{abstract}
Compositional simulations (neglecting gravity) of a simple reservoir element consisting of regions of high and low permeability reveal imbibition of oil into the low permeability region during depletion, and extraction of the oil from this region during lean gas injection by 'capillary pumping', a mechanism driven by variations in interfacial tension. Initial fluid composition is found to have a significant effect. The range of dimensionless capillary numbers for which capillary pressure may be neglected is determined, but evaluation of these numbers is not easy.
\end{abstract}

\section{Introduction}

There has been increasing recognition in recent years of the heterogeneous nature of oil and gas reservoirs, and geological detail at ever smaller scales is being included in finely-gridded reservoir models. Flow simulations are often conducted on these models, if not for full field simulation then as part of an upscaling procedure. Capillary pressure effects, which are often negligible at the reservoir scale, become increasingly important at smaller scales. Thus, at some stage it will become necessary to include capillary pressure in the flow simulations. Because this will significantly increase the computational time, it is important to know when it will be necessary.

Several previous studies $[1,2,3]$ have considered the interaction between capillary pressure and smallscale heterogeneity in waterflooding, but little attention has been paid to gas injection processes where compositional effects are important. In water-wet reservoirs, capillary pressure causes imbibition of water into regions of low permeability during waterflooding. By contrast, it inhibits the entry of gas into low permeability regions, since gas is not the wetting phase. It may thus accentuate the effect of heterogeneity and have an adverse effect on recovery. On the other hand, extraction of bypassed oil from low permeability regions may be accelerated by 'capillary pumping' $[4,5,6]$, a mechanism observed in recent experiments related to fractured systems (see Section 4.1 for a detailed description).

One study of gas/oil systems is that of Fayers and Lee [7], who performed simulations of lean and rich gas injection in a two-layer reservoir, either including or neglecting the capillary pressure and diffusion forces. They found these forces to be more significant in the rich gas case, with surprisingly little effect for the lean gas case. To some extent, our study follows on from their work in that we use a similar reservoir model. However, we consider a different phase behaviour model (i.e. different fluids) and a different recovery process, namely depletion followed by lean gas recycling.

\section{Simulation Model}

\subsection{Reservoir Geometry}

We consider a small $(10 \times 5 \mathrm{~m})$ two-dimensional areal model (Figure 1) of a low permeability region ( $k=10 \mathrm{mD}, \phi=0.20)$ surrounded by rock of higher permeability $(k=200 \mathrm{mD}, \phi=0.25)$. A grid of $15 \times$ 


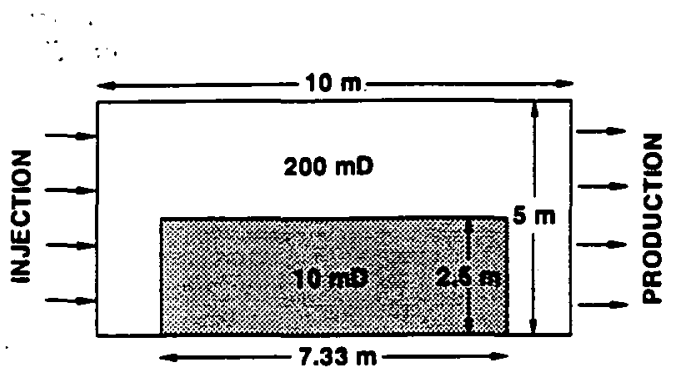

Figure 1: Reservoir Model

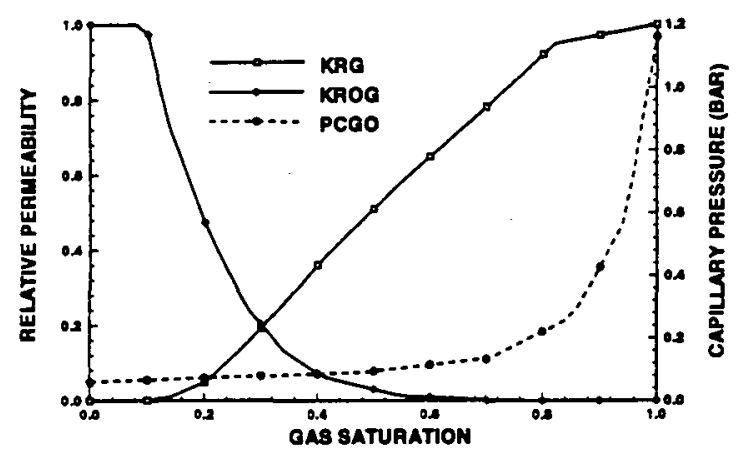

Figure 2: Relative Permeability and Capillary Pressure

10 blocks is used. Grid refinement tests have been performed to ensure that this is adequate.

\subsection{Rock Properties}

The relative permeability and capillary pressure curves used are shown in Figure 2. Hysteresis is neglected. The values of the capillary pressure shown in Figure 2 are multiplied for the simulation by: const $\sqrt{\phi / k}$. The constant is chosen such that this multiplicative factor is equal to one in the tight zone. For the permeable zone, smaller values are then obtained. Note that the relative permeability and capillary pressure curves are both functions of the interfacial tension (IFT) between the oil and gas phases, which in turn depends on the phase compositions and the pressure. For interfacial tensions below 1.5 $\mathrm{dyn} / \mathrm{cm}$, the capillary pressure is reduced in proportion to IFT, while the relative permeability curves are generally moved towards straight lines.

\subsection{Fluid Properties}

A 7-component equation of state model was used to describe the phase behaviour. Three different initial fluid compositions have been considered: Fluid $\mathrm{A}$ is a retrograde gas condensate, while Fluids B and C are volatile oils. Phase envelopes for the three fluids are shown in Figures 3-5. No water is present.

In all cases, a period of depletion was simulated (1

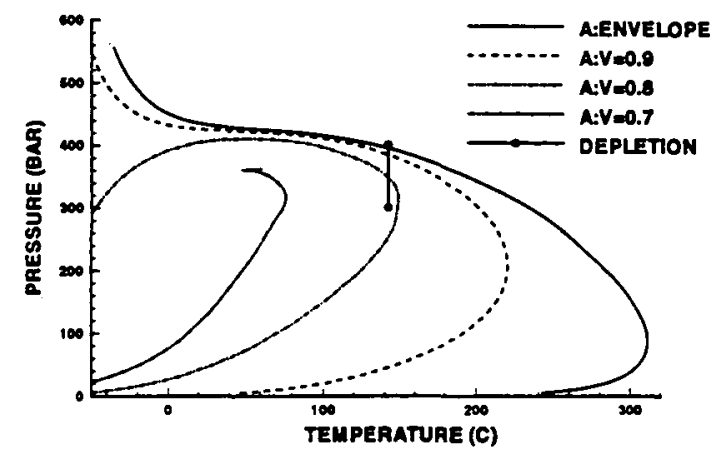

Figure 3: Phase Diagram for Fluid A

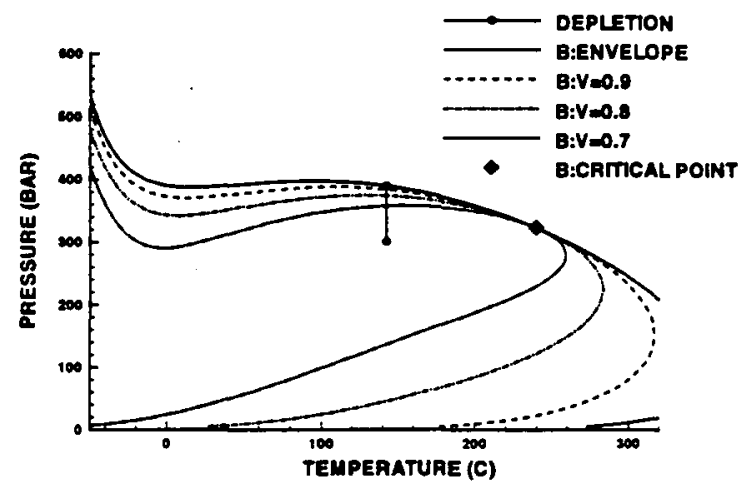

Figure 4: Phase Diagram for Fluid B

year), at the end of which the reservoir pressure was about 300 bar. This was followed by a period of lean gas injection (1 year). For Fluid A, the period of depletion allows a condensate saturation to develop: the liquid dropout curve for this fluid reaches about $27 \%$ at 300 bar. This condensate is partially revaporised during the period of lean gas injection. For Fluid B, whose bubble point is equal to the initial reservoir pressure of $389 \mathrm{bar}$, a high gas saturation develops during the depletion. A flash of this fluid at 300 bar gives a gas saturation of about $44 \%$. Fluid $\mathrm{C}$ has a lower bubble point ( 360 bar) and is less nearcritical in its behaviour, the gas saturation at 300 bar being only $21.7 \%$. For Fluids $B$ and $C$, lean gas injection recovers additional oil both by displacement and by vaporisation.

\subsection{Flow Rates}

To preserve the correct balance of the different forces, it is necessary to ensure that the flow rates are realistic. Our model is intended to represent a small portion of a heterogeneous reservoir, not situated close to a well. During the depletion period, fluid flows out of this portion of the reservoir towards the production well; at the same time, fluid flows into this portion from an adjacent portion further away from the production well. The composition of the fluid entering the portion modelled will be approximately the same as that of the fluid leaving it, but the flow rate will be slightly lower so that a gradual decrease 


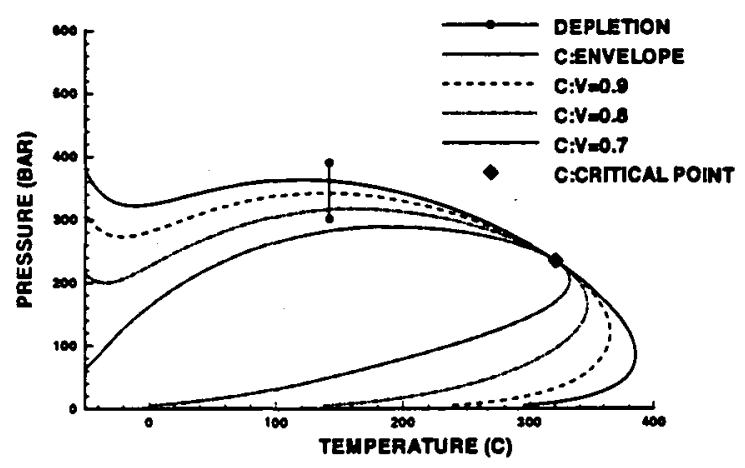

Figure 5: Phase Diagram for Fluid C

in pressure occurs. To model this situation, we reinject $98 \%$ of the produced fluids during the depletion period. This ensures that the depletion period has a reasonable length (one year) while maintaining a realistic average flow rate of about $0.2 \mathrm{~m} / \mathrm{d}$.

One difficulty caused by this approach is that the simulators we used only allow produced gas to be recycled. Thus, to reinject a proportion of the total fluid produced, we must adjust the separator specifications to assign all produced fluid to the gas phase. Surface liquid production is then calculated using split factors (determined by a flash calculation of the initial reservoir fluid at surface conditions) and component specific gravities, rather than from the EOS. Note that all liquid production figures quoted in this paper refer to surface liquid volumes, and are net figures adjusted for the fluid reinjected during the depletion period.

During the lean gas injection period, the injection rate is adjusted so that the frontal advance rate of the gas in the high permeability region is about 5 $\mathrm{m} /$ month. Injection is at a constant bottom-hole pressure of 300 bar. One year of lean gas injection is simulated, corresponding to almost three pore volumes of gas.

\section{Dimensional Analysis}

\subsection{Parameters}

Even this simple two-zone model, neglecting gravity and diffusion/dispersion, involves many parameters. Dimensional analysis of the governing equations [1] shows that the importance of the capillary pressure depends on the longitudinal and transverse capillary numbers:

$$
N_{C L}=\frac{K_{x} B_{c}}{L_{x}^{2} M Q} \quad . \quad N_{C T}=\frac{K_{y} B_{c}}{L_{y}^{2} M Q}
$$

where $B_{c}$ is a characteristic value of the slope of the capillary pressure curve. In addition to these two dimensionless numbers, the permeability ratio (strictly the ratio of permeability/porosity) between the two regions will influence the results.
Other parameters are the shapes of the relative permeability and capillary pressure curves and all the parameters in the phase behaviour model, which in turn determine the viscosity or mobility ratio of the lean gas flood. In this study, we assume fixed values for all these other parameters.

\subsection{Evaluation}

Evaluation of the dimensionless numbers is not straightforward. For example, the representative viscosity value, $M$, could be either the oil viscosity $(\approx$ $0.3 \mathrm{cP})$ or the gas viscosity $(\approx 0.03 \mathrm{cP})$. In the reservoir, it is mostly gas that is flowing and therefore it is the gas viscosity that determines the viscous pressure drop. On the other hand, segregation of the oil and gas under capillary or gravity forces cannot occur if the oil does not flow, and the rate of any such segregation will be controlled by the oil mobility.

Similarly, the evaluation of $B_{c}$ is difficult because the interfacial tension varies from $10^{-2} \mathrm{dyn} / \mathrm{cm}$ or lower near the saturation pressure through $0.3-0.8$ $\mathrm{dyn} / \mathrm{cm}$ at the end of the depletion period to 7.5 dyn $/ \mathrm{cm}$ during lean gas injection. Also, the slope of the capillary pressure curve varies from $\sim 0.03$ bar at low gas saturation through $\sim 0.5$ bar at intermediate gas saturation to $\sim 5.2$ bar at high gas saturation, as well as by a factor of 4 between the two regions.

For now, we use the oil viscosity, the reference interfacial tension of $1.5 \mathrm{dyn} / \mathrm{cm}$, the capillary pressure slope at intermediate gas saturations in the tight zone, and an arithmetic average of the permeabilities in the two regions, finally obtaining:

$$
N_{C L} \approx 2 \quad N_{C T} \approx 8 .
$$

The permeability ratio is $20: 1$.

\section{Effect of Capillary Pressure}

\subsection{Fluid B}

The evolution of the oil saturation over the simulated production time of two years for Fluid B is shown in Figure 6. Quite complex saturation patterns are observed.

During depletion, capillary pressure expels gas from the low permeability region and imbibes oil into this region. This process is maintained as the pressure drops by the increasing interfacial tension which leads to increasing capillary pressure.

During lean gas injection the low viscosity of the gas means that the gas pressure is almost constant, so that the oil pressure is lowest where the capillary pressure is greatest. Figure 7 shows the capillary pressure at $\mathbf{4 2 5}$ days: it is greatest near the injection well, and greater in the high permeability re- 
100 DAYS

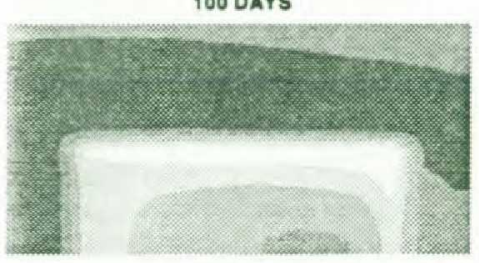

300 DAYS

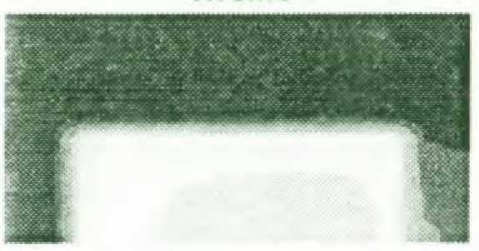

425 DAYS

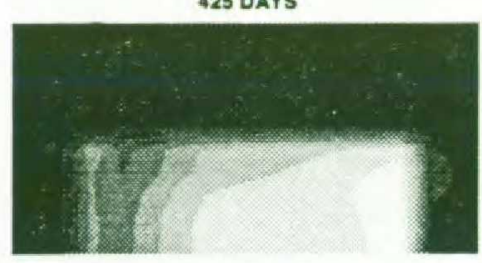

395 DAYS

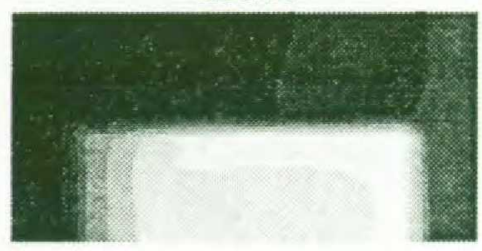

500 DAYS

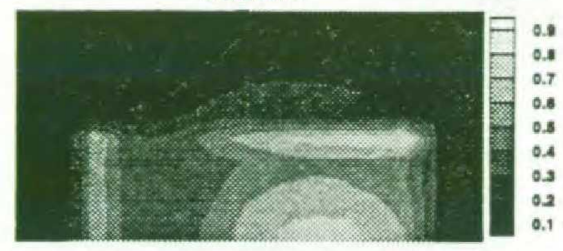

700 DAYS

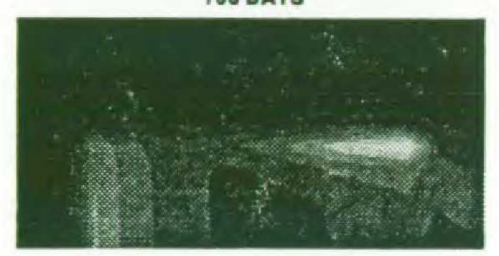

Figure 6: Oil Saturation Evolution, Fluid B

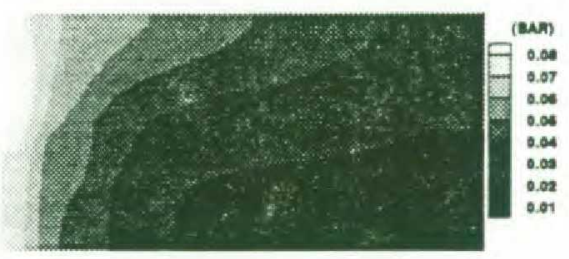

Figure 7: Capillary Pressure at 425 days, Fluid B

gion than in the low permeability region. This is because the variations in capillary pressure are controlled mainly by the variations in interfacial tension, which is greater between the oil and the injected gas than between the oil and its equilibrium gas (especially after the intermediate components have been vaporized). Thus, oil flows backwards, away from the production well towards the injection well, i.e. in the opposite (counter-current) direction to the gas. Also, oil flows out of the low permeability region into the high permeability one.

This 'reverse imbibition' is similar to the 'capillary pumping' mechanism observed experimentally for fractured systems $[4,5,6]$, but differs in that it is purely a capillary pressure effect with no involvement of diffusion. In the experiments, diffusion of lean gas into the matrix means that the gas is leanest near the fracture, which in turn means the capillary pressure is strongest there and this causes the oil to be sucked towards the fracture where it can be extracted into the gas flowing through the fracture by diffusion. In our problem, the existence of capillary pressure in the high permeability rock means that the capillary pumping could move oil from the interior of the low permeability region, not only to the interface with the high permeability region but right out into it under the influence of capillary forces alone.

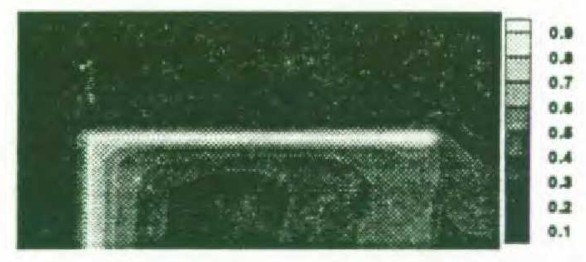

Figure 8: Oil Saturation at 700 days, Fluid B $30 \times 20$ block grid

At the end of the flood, two thin streaks of high oil saturation remain just inside the tight zone, one along the face near the injection well, the other at the lateral boundary with the permeable zone near the production well. It is not clear why the oil saturation should remain high in these places, because the capillary pressure gradient at these boundaries should cause the oil to flow out into the permeable zone. In a grid refinement test (Figure 8), the streaks became even thinner but more pronounced. The fact that they are essentially one grid block thick on both grids might suggest a numerical artefact, but the same results have been obtained both with MORE and with ECLIPSE-300, the latter in both IMPES and fully implicit modes. At present, no explanation for the existence of these streaks has been found.

Figure 9 and Table 1 show the surface liquid production with and without capillary pressure. During the depletion period (the first year), capillary pressure effects suck oil into the tight zone, reducing liquid production (by $6.4 \%$ in this case). During lean gas injection, the 'capillary pumping' competes with the increased tendency for gas to bypass the tight zone due to the higher initial oil saturation there, but the net effect is in fact a reduction (by $8.8 \%$ ) in the liquid recovery in comparison to the case without capillary 
Table 1: Liquid Recovery, Fluid B

\begin{tabular}{|l|c|c|}
\hline \multirow{2}{*}{} & \multicolumn{2}{|c|}{ Recovery (\%OIP) } \\
\cline { 2 - 3 } & 1 Year & 2 Years \\
\hline \hline With $\mathrm{P}_{c}$ & 7.70 & 56.55 \\
No $\mathrm{P}_{c}$ & 8.23 & 61.78 \\
Homogeneous, No $\mathrm{P}_{c}$ & 8.23 & 70.80 \\
\hline
\end{tabular}

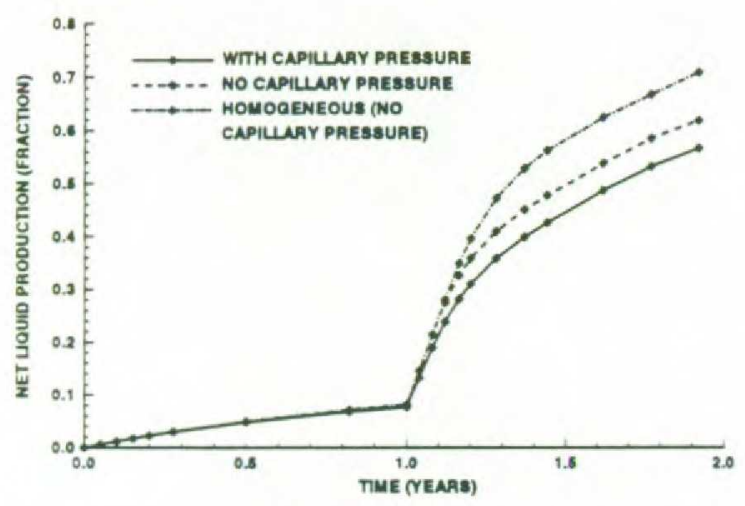

Figure 9: Liquid Production, Fluid B

pressure. Also shown in Figure 9 and in Table 1 is the case of a homogeneous reservoir with the same average properties and no capillary pressure. This case shows that the heterogeneity has no effect on recovery during depletion if capillary pressure is neglected, and that the reduction in recovery during lean gas injection due to the heterogeneity is $12.6 \%$ if capillary pressure is neglected but $21.9 \%$ if it is included.

\subsection{Fluid C}

For Fluid C, the less volatile oil, the evolution of the oil saturation is qualitatively similar to that for Fluid $\mathrm{B}$, though the saturation patterns observed are not quite so complicated (Figure 10). Because the oil is less volatile, less gas evolves during the depletion period. Oil is again imbibed from the permeable zone into the tight zone, where the oil saturation remains very high. During lean gas injection, this high oil saturation delays the entry of the injected gas into the tight zone. Only towards the end of the flood does a significant amount of gas enter this zone. At his time, the flow of oil is again backwards towards ne injection well and outwards from the tight zone into the permeable zone.

The effect of the capillary pressure on liquid recovery is greater for Fluid C than for Fluid B (Figure 11). It reduces the liquid production during depletion and lean gas injection by $12.4 \%$ and $13.9 \%$ respectively, the overall reduction being $13.6 \%$. The heterogeneity again has no effect on recovery during depletion if capillary pressure is neglected, but it reduces re-
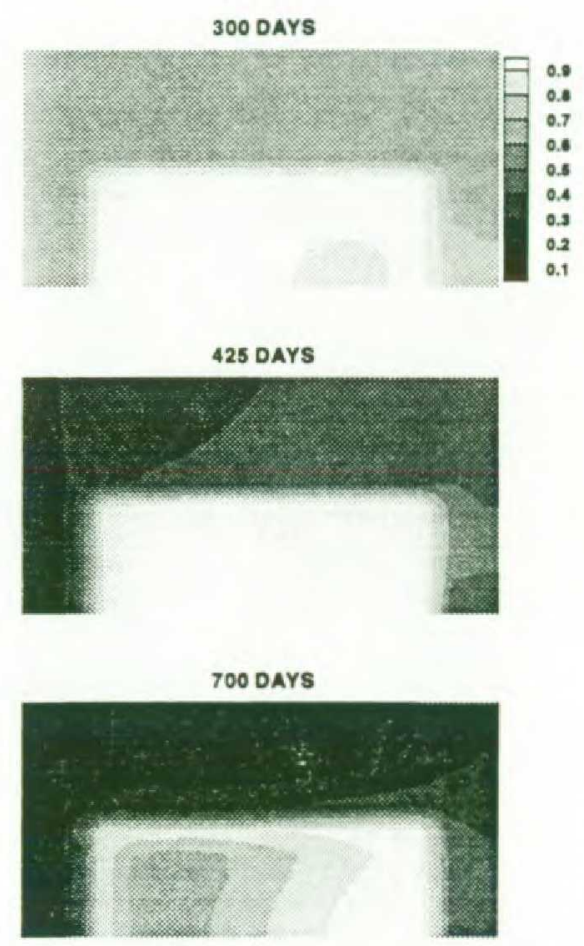

Figure 10: Oil Saturation Evolution, Fluid C

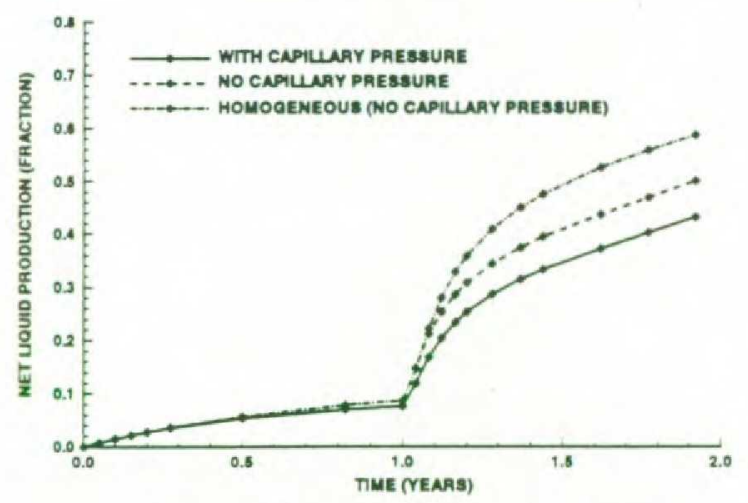

Figure 11: Liquid Production, Fluid C

covery during lean gas injection by $17.3 \%$ if capillary pressure is neglected and by $28.8 \%$ if capillary pressure is included.

\subsection{Fluid A}

For the gas condensate Fluid A, the oil saturations are much lower for than for the two volatile oils. Nonetheless, imbibition of condensate into the tight zone does occur during the depletion, and some of the same oil saturation patterns occur during lean gas injection (Figure 12). However, the effect of capillary pressure on liquid production is negligible for Fluid A. 


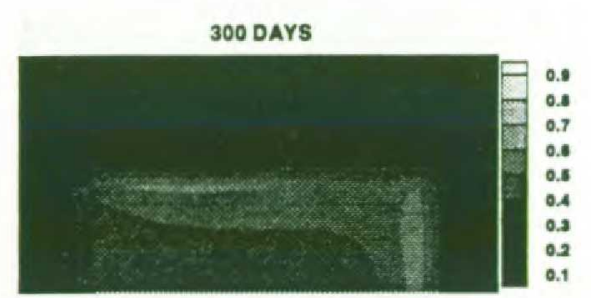

500 DAYS

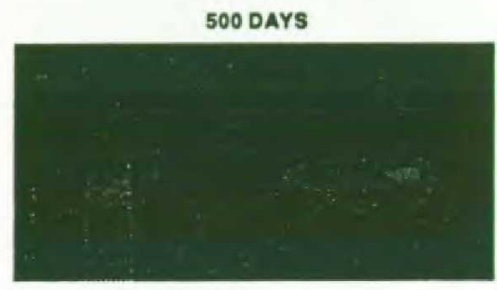

Figure 12: Oil Saturation Evolution, Fluid A

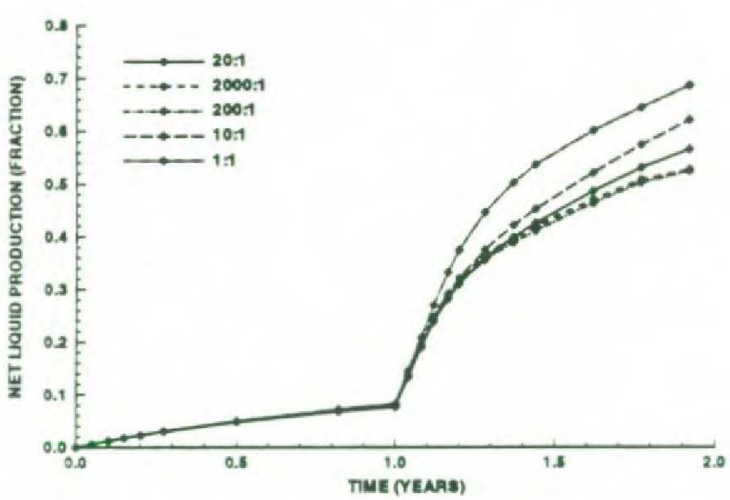

Figure 13: Effect of Permeability Ratio Fluid B (with Capillary Pressure)

\section{Quantification}

\subsection{Effect of Permeability Ratio}

The effect of increasing the permeability ratio between the permeable and the tight zones has been investigated for Fluid B. The ratio was varied keeping the average permeability constant. The effect on the liquid production is shown in Figure 13 and Table 2.

During depletion, the liquid production is a minimum for the original permeability ratio of $20: 1$. Further increases in this ratio actually increase liquid recovery. Although the capillary pressure in the tight zone becomes greater as the permeability ratio is increased, the lower permeability there restricts the amount of oil that can be imbibed into the tight zone. A region of high oil saturation builds up just inside the tight zone, as can be seen for the 2000:1 ratio case in Figure 14. This oil flows very slowly into the tight zone, and prevents much further oil from being imbibed. During lean gas injection, liquid re-
Table 2: Effect of Permeability Ratio, Fluid B

\begin{tabular}{|c|c|c|}
\hline Permeability & \multicolumn{2}{|c|}{ \%OIP Liquid Production } \\
\cline { 2 - 3 } Ratio & 1 Year & 2 Years \\
\hline \hline $1: 1$ & 8.22 & 68.59 \\
$10: 1$ & 7.83 & 62.04 \\
$20: 1$ & 7.70 & 56.55 \\
$200: 1$ & 7.77 & 52.45 \\
$2000: 1$ & 7.93 & 52.70 \\
\hline
\end{tabular}

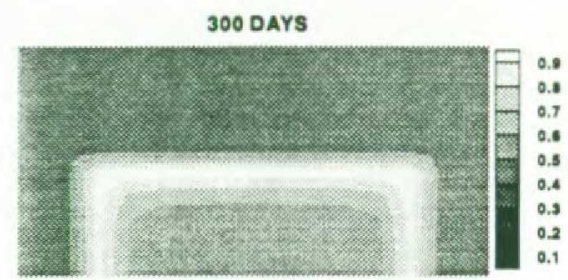

700 DAYS

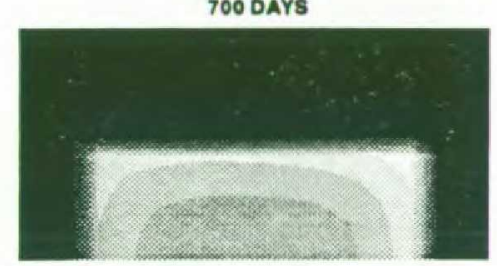

Figure 14: Oil Saturation, Permeability Ratio 2000:1 Fluid B

covery decreases as the permeability ratio increases from 1 to 200 but thereafter is approximately constant. Very little liquid is recovered from the tight zone by lean gas injection when the permeability ratio is this high (see Figure 14), again because of the very low oil flow rates that are possible in the tight zone.

\subsection{Effect of Capillary Numbers}

In this section, the effect of varying the length and width of the reservoir, as well as the strength of the capillary pressure, has been investigated. A permeability ratio of 20:1 was used in all cases. Initially, the reservoir proportions (length:width ratio) were altered, keeping the total reservoir volume constant. The cases considered are shown in Table 3. Case

Table 3: Reservoir Proportions

\begin{tabular}{|c|c|c|c|c|}
\hline Case & $L_{x}(m)$ & $L_{y}(m)$ & $N_{C L}$ & $N_{C T}$ \\
\hline \hline 1 (Original) & 10 & 5.0 & 2.0 & 8 \\
2 & 31.62 & 1.58 & 0.2 & 80 \\
3 & 100 & 0.5 & 0.02 & 800 \\
4 & 3.16 & 15.8 & 20.0 & 0.8 \\
\hline
\end{tabular}


2 represents an increase by a factor of $\sqrt{10}$ in the length and a decrease by $\sqrt{10}$ in the width by comparison with the original reservoir, Case 1 . Since the capillary numbers are proportional to the square of the reservoir dimensions, the stretched reservoir has a longitudinal capillary number 10 times smaller than the original reservoir and a transverse one 10 times larger. Case 3 represents a further stretching of the reservoir by the same factors, while Case 4 is shorter and wider.

Table 3 also shows the longitudinal and transverse capillary numbers for each case assuming the level of capillary pressure described in Section 2.2. Runs have also been made with the strength of the capillary pressure increased or decreased by one or more factoirs of 10 .

Some additional runs were also made in which the length of the reservoir was increased, keeping the width constant. This changes the total pore volume of the reservoir, and the imposed flow rates must be changed in proportion to the total pore volume to keep the length of the flood and, more importantly, the transverse capillary number, unchanged. The longitudinal capillary number is then inversely proportional to the square of the reservoir length.

There is a limit to the extent to which the reservoir length can be increased while still maintaining some comparability with other cases. Once the reservoir becomes too long, the viscous pressure drop between the injector and producer becomes large enough to significantly alter the phase behaviour, and the results can no longer be interpreted simply in terms of the capillary numbers. In our case, the viscous pressure drop is still negligible (in phase behaviour terms) when the reservoir is $100 \mathrm{~m}$ long, but has reached 8 bars, which is no longer negligible for these near-critical fluids, at $316 \mathrm{~m}$.

The results of many runs are summarised in Figures 15,16 and 17 . These plots show the percentage reduction in the liquid production after two years, compared to the reference case of a homogeneous reservoir with no capillary pressure. This quantity is plotted as a function of $N_{C L}$ and $N_{C T}$ for the 3 different fluids. Each data point in these figures represents the result of a single simulation (except where grid refinement tests were performed to confirm the adequacy of the grid of $15 \times 10$ blocks that was used). Only one combination of the capillary numbers was obtained in two different ways: first using reservoir Case 2 with the capillary pressure reduced by a factor of 10 , second using reservoir Case 1 with the standard capillary pressure and the reservoir length increased by a factor of 10 . For Fluid B, the losses in recovery were $16.3 \%$ and $16.6 \%$ respectively, and the liquid production curves were almost identical.
ReLATIVE NET LOSSES IN THE LUOUID PROOUCTON (x)

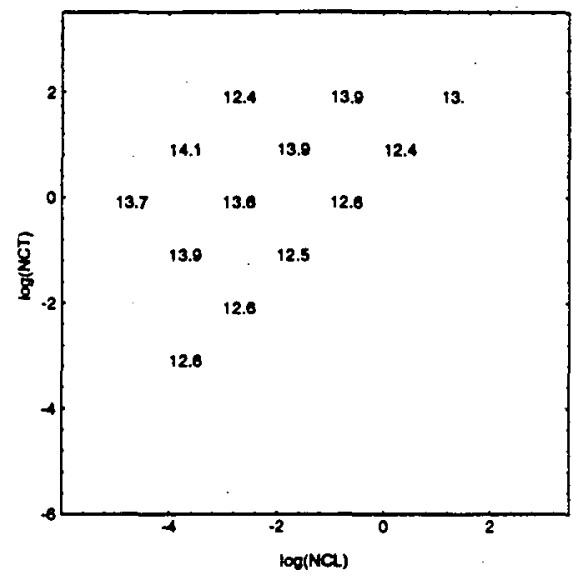

Figure 15: Loss of Liquid Production, Fluid A

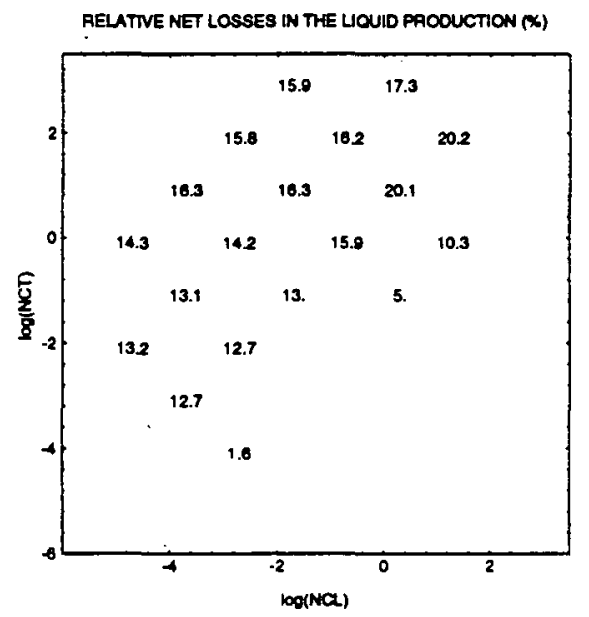

Figure 16: Loss of Liquid Production. Fluid B

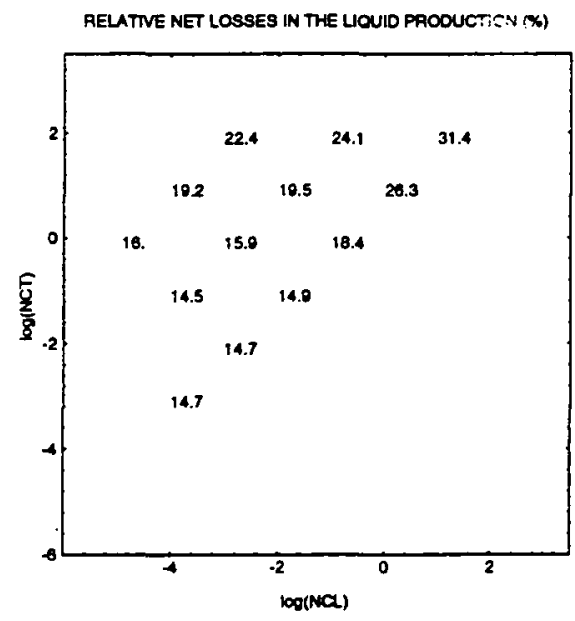

Figure 17: Loss of Liquid Production, Fluid C 


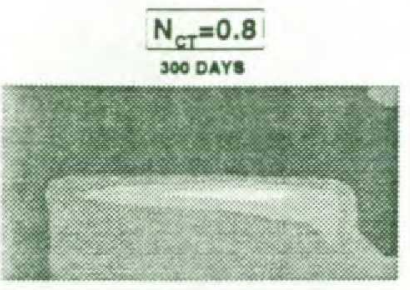

425 DAYs

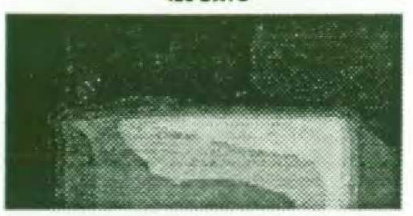

700 DAYs

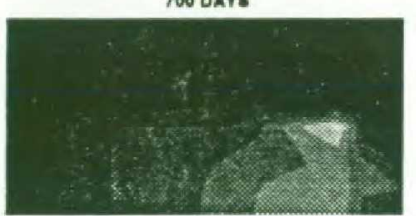

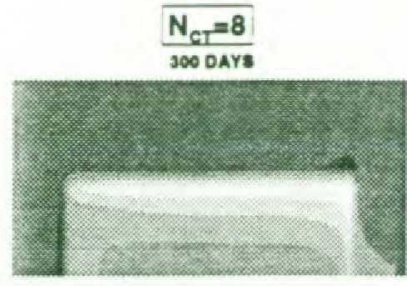

425 DAYs

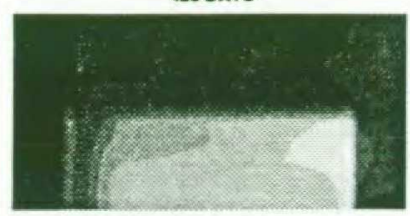

700 DAYs

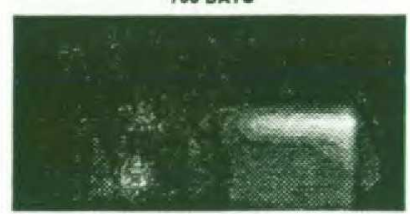

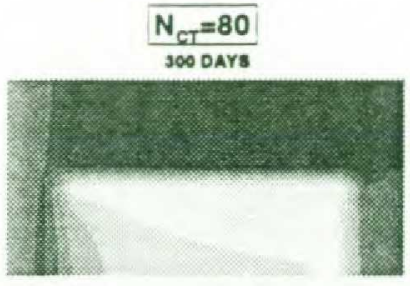

425 DAYs

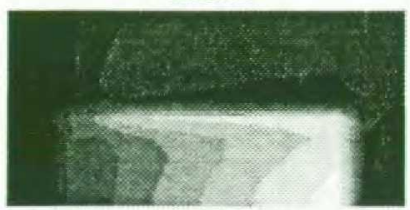

700 DAY:

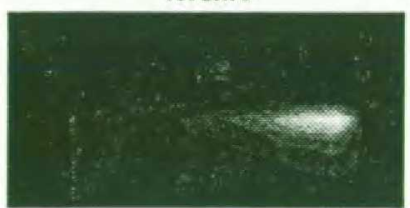

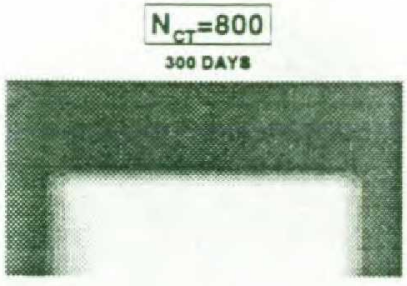

425 Dar:

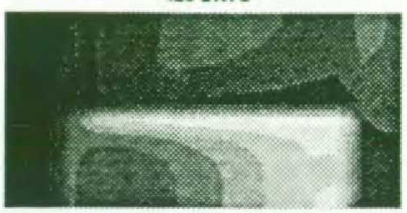

700 DAYS

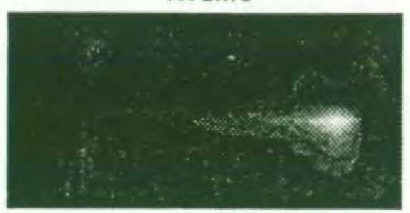

Figure 18: Effect of $N_{C T}$ on Oil Saturation Patterns at $N_{C L}=0.2$, Fluid B Scale same as Figure 6

\subsubsection{Interpretation for Fluid A}

For Fluid A (Figure 15), the capillary pressure has very little effect on liquid production over the whole range of capillary numbers that has been investigated. This is probably because the condensate saturation is not high enough to allow significant flow of the liquid phase within the reservoir:

\subsubsection{Interpretation for Fluid C}

For Fluid C (Figure 17), there is increasing loss of liquid production as the capillary numbers increase. For longitudinal capillary numbers less than about 0.2 , the capillary pressure starts to becomes significant at around $N_{C T} \approx 1$. For $N_{C L}=2$ or more, longitudinal capillary pressure effects also reduce liquid recovery. Recovery continues to decline as th capillary numbers increase.

\subsubsection{Interpretation for Fluid B}

For Fluid B (Figure 16), the picture is more complicated. For longitudinal capillary numbers less than about 0.2 , the capillary pressure again comes into play at around $N_{C T} \approx 1$, but the impact on the net liquid production is not very great. In fact, the loss reaches a maximum of around $16-17 \%$ at $N_{C T}=8$ and decreases slightly as $N_{C T}$ increases further.

This behaviour can be explained in terms of the 'capillary pumping' mechanism described in Section 4.1, and is illustrated in Figure 18 which shows the saturation patterns which develop in a series of cases with (almost) constant $N_{C L}$ and increasing $N_{C T}$. During depletion, the thickness of the region of high oil saturation within the tight zone increases as $N_{C T}$ increases, as would be expected. During lean gas injection at $N_{C T}=0.8$, the capillary pressure plays little role. The oil remaining at the end of the flood is distributed throughout the tight zone at a relatively low saturation. At higher $N_{C T}$, the 'capillary pumping' mechanism draws the oil from the tight zone towards the permeable zone. This effect increases with increasing $N_{C T}$. The oil remaining at the end of the flood occurs mainly in the narrow streak just inside the boundary of the tight zone. At $N_{C T}=8$, there is remaining oil both in this streak and elsewhere in the tight zone, so the loss in liquid production has increased. For higher $N_{C T}$, however, the capillary pressure has removed the oil from the rest of the tight zone, so only that in the streak remains, so the loss decreases again.

Moving to higher $N_{C L}$, the essential differences in the saturation patterns can be seen by comparing the original case described in Section 4.1 and Figure 6 with the $N_{C T}=8$ case in Figure 18. These two cases differ only in that the original case has $N_{C L}=2$ while the other case has $N_{C L}=0.2$. For the case with higher $N_{C L}$, high oil saturation occurs during depletion at the inlet end of the tight zone, and a thin streak of high oil saturation remains there after lean gas injection. The presence of this second thin streak of remaining oil accounts for the larger loss of liquid production seen in Figure 16 for the cases with $\left(N_{C L}, N_{C T}\right)=(2,8)$ (the original case) and with $\left(N_{C L}, N_{C T}\right)=(20,80)$ (the original case but with higher capillary pressure). In 


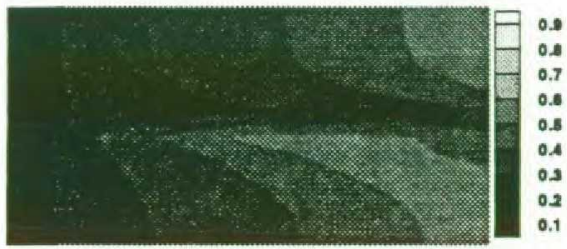

Figure 19: Oil Saturation, $3.16 \times 15.8 \mathrm{~m}$ Reservoir Fluid B, No Capillary Pressure

the case with $\left(N_{C L}, N_{C T}\right)=(2,800)$, the stronger 'capillary pumping' effect partially offsets the loss in recovery due to the second thin oil streak.

Finally, for the shorter but wider reservoir Case 4, the anisotropy number [1]:

$$
R_{x y}=\sqrt{\frac{K_{y}}{K_{x}}} \frac{L_{x}}{L_{y}}=\sqrt{\frac{N_{C T}}{N_{C L}}}<1
$$

which means that the reservoir is no longer in socalled 'VE'1 [8]. Consequently the reservoir behaves as though it were homogeneous, except close to the interface between the two zones, as can be seen in Figure 19. The loss of liquid production due to the heterogeneity is thus much lower, but, as for the other cases, it starts to increase when $N_{C L} \approx 1$ or greater.

\subsubsection{Discussion}

For all three fluids, capillary pressure seems to be negligible provided both $N_{C T}$ and $N_{C L}$ are significantly less than 1 . When either capillary number $\approx 1$ or greater, the impact of capillary pressure on liquid production depends on the initial fluid composition. These results suggest that our definition of the capillary numbers is a reasonable one, despite the difficulties discussed in Section 3.2.

Despite having modelled a completely different process (waterflooding in water-wet reservoirs), the results of Yokoyama and Lake [1] are similar to ours in that for a model with $N_{C L}=4 \times 10^{-3}$, the transition from negligible to dominant capillary pressure occurs for $10^{-1}<N_{C T}<10^{1}$, while for a one-dimensional case $\left(N_{C T}=\infty\right)$, longitudinal capillary pressure effects begin to appear at $N_{C L} \approx 10^{-1}$.

\subsection{Effect of $k_{r}(\sigma)$ model}

Because of the unusual features observed in the simulations with strong capillary pressure, and because some convergence difficulties were experienced

\footnotetext{
${ }^{1} \mathrm{VE}$ stands for 'vertical equilibrium', though in our case this is a misnomer since no gravity is involved and 'transverse equilibrium' would be a better term.
}

Table 4: $k_{r}(\sigma)$ Models

\begin{tabular}{|c|l|c|c|}
\hline \multirow{2}{*}{ Model } & \multirow{2}{*}{} & \multicolumn{2}{|c|}{$\begin{array}{c}\text { \%OIP Liquid } \\
\text { Production }\end{array}$} \\
\cline { 3 - 4 } & Description & Year & 2 Years \\
\hline \hline A & Original model & 7.70 & 56.55 \\
B & Modified model & 7.31 & 54.33 \\
C & No dependence & 6.92 & 53.80 \\
\hline
\end{tabular}

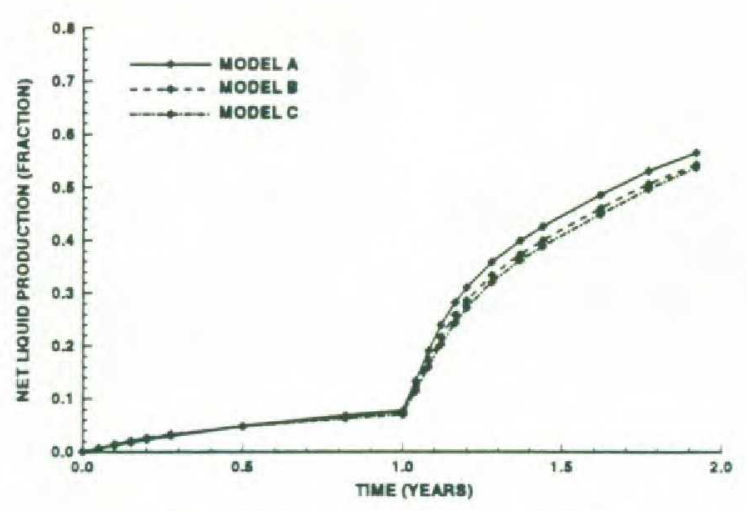

Figure 20: Effect of $k_{r}(\sigma)$ Model

Fluid B, with Capillary Pressure

in obtaining these results, three different simulators (MORE, ECLIPSE-300 and COMP-4) were used to confirm the accuracy of the results and to find the most efficient simulator for the job.

Unfortunately, the treatment of the dependence of relative permeability $\left(k_{r}\right)$ on interfacial tension $(\sigma)$ in each of the three simulators is different. Although we were able to adapt one of the simulators so as to confirm that all three models gave the same results when posed with the same problem, we did make runs with the three different $k_{r}(\sigma)$ models shown in Table 4. Here, the modified model differs from the original model only in the way the interpolation between high and low interfacial tension rel perm tables is performed. All three models treat the capillary pressure in the same way, as a linear function of the interfacial tension.

Figure 20 and the Table 4 show that changing the $k_{r}(\sigma)$ model has a significant effect on the liquid production during depletion (for the case of Fluid B with capillary pressure included). Liquid production during depletion is reduced by $5.1 \%$ by using Model B, and by $10.2 \%$ by using Model C. This happens because the oil mobility at low interfacial tensions is reduced (more so in Model C than Model B), leading to reduced oil flows at a given gas saturation. The reduction in liquid recovery during lean gas injection is less dramatic, $3.7 \%$ for Model B and $4.0 \%$ for Model C.

Despite these differences in liquid recovery, the saturation patterns observed were qualitatively similar 
with all three models.

\subsection{Computational Aspects}

From a computational point of view, compositional simulation of capillary-dominated problems presents a severe challenge for current reservoir simulators. Where either capillary number was more than about 10 , the simulations could take a day or more on an HP 750 workstation, since very short timesteps were required. However, the IMPES approach was found to remain more efficient than fully implicit, despite the very severe timestep restrictions.

\section{Conclusions}

We have considered the effect of capillary pressure on depletion and lean gas injection in a simple heterogeneous reservoir element consisting of regions of high and low permeability. The main conclusions are:

1. The effect of capillary pressure is negligible provided both the transverse and longitudinal capillary numbers are significantly less than 1 .

2 . When either capillary number $\approx 1$ or greater, the impact of capillary pressure on liquid production depends on the initial fluid composition. The dependence of relative permeability on interfacial tension can also have a significant effect, but the permeability ratio has relatively little effect.

3. For the gas condensate fluid studied, capillary pressure was found to have relatively little impact on the recovery process.

4. In cases where the initial fluid is a volatile oil and capillary forces are dominant, the flow of oil during lean gas injection is controlled by variations in capillary pressure with interfacial tension: 'reverse imbibition' or 'capillary pumping' of orl from low to high permeability regions can occur, but the recovery efficiency is limited by (unexplained) streaks of high oil saturation which remain just inside the tight zone at the end of the flood.

5. Evaluation of the capillary numbers is not easy, because of the need to choose representative values of quantities which exhibit large variations during the recovery process.

Much further work is needed to fully understand the role of small-scale heterogeneities in high pressure gas flooding. In our study, we have neglected gravity. In fact, this would be the dominant force for a vertical reservoir element of the size we considered, but capillary pressure becomes increasingly significant as the thickness of the model decreases and would be dominant in a laminated rock [3], for example. We have also neglected diffusion and dispersion, which could be significant at small scales. Also, because of the large number of parameters associated with the phase behaviour, caution should be used in extrapolating the results from this study to other reservoir fluids. Finally, in the cases where capillary pressure and diffusion/dispersion cannot be neglected, the implications for upscaling procedures have not yet been investigated.

\section{Nomenclature}

$\begin{array}{ll}B_{c} & \text { characteristic value of slope (derivative) of } \\ & \text { capillary pressure curve } \\ k & \text { permeability } \\ K_{x} & \text { characteristic value of } x \text {-direction permeability } \\ K_{y} & \text { characteristic value of y-direction permeability } \\ L_{x} & \text { characteristic length in } x \text {-direction } \\ L_{y} & \text { characteristic length in y-direction } \\ M & \text { characteristic value of viscosity } \\ N_{C L} & \text { longitudinal capillary number (dimensionless) } \\ N_{C T} & \text { transverse capillary number (dimensionless) } \\ P_{c} & \text { capillary pressure } \\ Q & \text { characteristic value of flow rate } \\ R_{x y} & \text { anisotropy number (dimensionless) } \\ \sigma & \text { interfacial tension } \\ \phi & \text { porosity }\end{array}$

\section{References}

[1] Y. Yokoyams and L.W. Lake, 'The Effects of Capillary Pressure on Immiscible Displacements in Stratified Porous Media', SPE 10109, SPE Ann. Tech. Conf. Exinib., San Antonio, October 1981.

[2] T.F.M. Kortekaas, 'Water-Oil Displacement Characteristics in Cross-Bedded Reservoir Zones'. SPEJ, December 1985

[3] P.S. Ringrose, K.S. Sorbie, F.A. Feghi, G.E. Pickup and J.L. Jensen, 'Relevant Reservoir Characterisation: Recovery process; Geometry and Scale', $6^{\text {th }}$ Euro. Symp. on IOR, Stavanger, Norway, 21-23 May 1991.

[4] D.D. Morel, B. Bourbiaux, M. Latil and B. Thiebot, 'Diffusion Effects in Gas Flooded Light Oil Fractured Reservoirs', SPE 20516, SPE Ann. Tech. Conf. Exhib., New Orleans, October 1990, and SPE Advanced Technology Series, Vol. 1 No. 2, July 1993, pp 100-109.

[5] J-F.X. Le Romancer, D.F. Defives and G. Fernandes, 'Mechanism of Oil Recovery by Gas Diffusion in Fractured Reservoirs in the Presence of Water', SPE 27746, SPE/DOE $9^{\text {th }}$ Symp. on IOR, Tulsa, 17-20 April 1994.

[6] H. Hu, C.H. Whitson and Y. Qi, 'A Study of Recovery Mechanisms in a Nitrogen Diffusion Experiment', $6^{\text {th }}$ Euro. Symp. on IOR, Stavanger, May 1991.

[7] F.J. Fayers and S. T. Lee, 'Cross Flow Mechanisms in Oil Displacement by Gas Drive in Heterogeneous Reservoirs', SPE 24934, SPE Ann. Tech. Conf. Exhib., Washington D.C., October 1992.

[8] Y.C. Yortsos, 'A Theoretical Analysis of Vertical Equilibrium Flow', SPE 22612, SPE Ann. Tech. Conf. Exhib., Dallas, October 1991. 